\title{
Convolutional neural networks for the classification of chest X-rays in the loT era
}

\author{
Khaled Almezhghwi $^{1}$ (D) . Sertan Serte ${ }^{2}$. Fadi Al-Turjman ${ }^{3,4}$
}

Received: 12 May 2020 / Revised: 19 March 2021 / Accepted: 1 April 2021 /

Published online: 17 June 2021

(C) The Author(s), under exclusive licence to Springer Science+Business Media, LLC, part of Springer Nature 2021

\begin{abstract}
Chest X-ray medical imaging technology allows the diagnosis of many lung diseases. It is known that this technology is frequently used in hospitals, and it is the most accurate way of detecting most thorax diseases. Radiologists examine these images to identify lung diseases; however, this process can require some time. In contrast, an automated artificial intelligence system could help radiologists detect lung diseases more accurately and faster. Therefore, we propose two artificial intelligence approaches for processing and identifying chest X-ray images to detect chest diseases from such images. We introduce two novel deep learning methods for fast and automated classification of chest X-ray images. First, we propose the use of support vector machines based on the AlexNet model. Second, we develop support vector machines based on the VGGNet16 method. Combined deep networks with a robust classifier have shown that the proposed methods outperform AlexNet and VGG16 deep learning approaches for the chest X-ray image classification tasks. The proposed AlexNet and VGGNet based SVM provide average area under the curve values of $98 \%$ and $97 \%$, respectively, for twelve chest $\mathrm{X}$-ray diseases.
\end{abstract}

Keywords Convolutional neural networks · Deep learning

\section{Introduction}

The Internet of things (IoT) allows the connection of X-ray machines in hospitals for the purpose of collecting big data. All hospitals contain X-ray machines, and these machines

Khaled Almezhghwi

khaled.almezhghwi@neu.edu.tr

1 Electrical and Electronic Engineering, College Of Electronic Technology, Tripoli, Libya

2 Electrical and Electronic Engineering, Near East University, Nicosia, North Cyprus via Mersin 10, Turkey

3 Artificial Intelligence Department, Near East University, Nicosia, North Cyprus via Mersin 10, Turkey

4 Research Center for AI and IoT, Near East University, Nicosia, North Cyprus via Mersin 10, Turkey 
can be connected using IoT methods. Accessing all X-ray images means that massive amounts of data are produced. Then, this enormous amount of X-ray images can be modelled using artificial intelligence algorithms. Consequently, each hospital can connect to this central unit for the automated detection of diseases from X-ray images. Therefore, the IoT is a crucial tool in terms of the ability to instantly access data, and artificial intelligence is essential for modelling which enables diseases to be accurately detected on images. This work shows that the precise modelling of disease on X-ray images is possible by using deep learning models. Then this work will be extended for the multi-hospital setting.

Early treatment is crucial for people, as delays in treatment application can sometimes be fatal. Chest X-ray imaging enables the detection of pneumonia and other chest diseases. Figure 1 shows examples of healthy and pneumonia $\mathrm{X}$-ray images.

Deep learning techniques $[6,9,13,31-33]$ have been utilized for object detection in images. ImageNet challenge [3] has shown that these deep learning techniques, which are mainly convolutional neural networks, can sometimes even provide better classification performance than humans. Among these techniques/models, ResNet-152 provides the best object recognition performances compared to other learning methods. Other deep convolutional neural networks include AlexNet [13], VGG [31], GoogleNet [32, 33], ResNet [6] and DensNet [9].

These models have also been applied to medical datasets [10, 11, 18, 19, 23-25]. These models allow the modelling of medical data for the detection and recognition of diseases. Serte and Demirel [23] proposed the Gabor wavelet-based deep learning method for the recognition of malignant melanoma and seborrheic keratosis skin lesions. This method decomposed the skin image into seven directional Gabor bands and then modelled each of the representations using AlexNet and ResNet-18 deep learning models. Furthermore, Serte and Demirel [23] utilized a wavelet-based technique for the detection of malignant melanoma and seborrheic keratosis skin lesions. Their approach is based on obtaining skin image into wavelet band and then modelling each image band using deep networks. Authors [20, 27] have also utilized CNNs for detecting COVID-19 on X-Ray images, while others $[21,28]$ have also employed CNNs to identify COVID-19 on CT-scans. Furthermore, authors $[16,17,22,26,29]$ have shown that CNN models provide accurate results

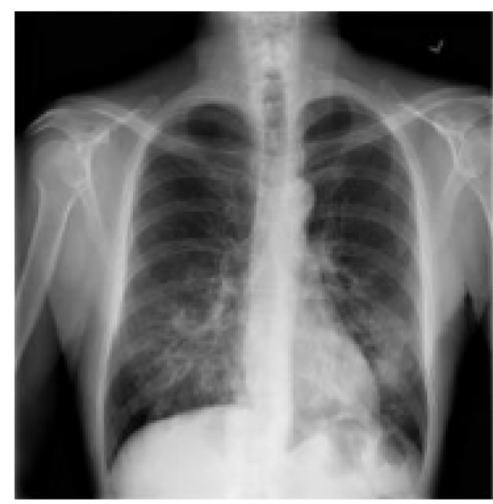

(a) Normal

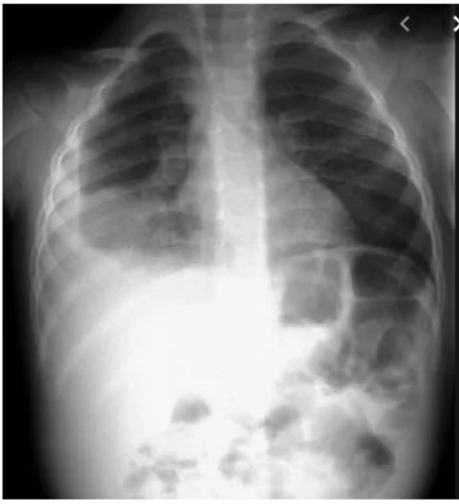

(b) Pneumonia

Fig. 1 Normal (a) and Pneumonia (b) cases on Chest X-ray images. Images are taken from the Chest X-ray 14 dataset [36] 
for eye disease. Therefore, CNN models can be used on different medical images for the diagnosis of disease types. A recent medical review paper [30] summarized the application of these models.

Recently, Jakub et al. [4] a convolutional neural network for classification of healthy, bacterial pneumonia, and viral pneumonia using X-ray images.

The proposed support vector machine-based AlexNet and VGG-16 models are different than the previously proposed AlexNet and VGG-16 models. AlexNet and VGG-16 models employ convolution, and the image representations are classified using a Softmax classifier. However, the proposed methods convolve images and then organize the convolution output using a support vector machine classifier (SVM). SVM is known as a more robust classifier than the Softmax classifier. As a result, using SMV in conjunction with AlexNet and VGG16 improves chest X-ray image classification performance.

The main contributions of this work are as follows. First, we propose a novel support vector machine-based AlexNet deep learning model for chest X-ray classification. This method builds on convolving images using AlexNet architecture for feature modelling and then classifying these features using support vector regressions. Second, we propose a novel support vector machine based VGG-16 deep learning model for chest X-ray image classification. This method builds on convolving images using the VGG-16 model to obtain image representations and then learns these representations using support vector regressions.

The paper organizes the work in the following sections. First, related work provides a summary of the previous studies on skin lesion classification. Second, the proposed novel techniques are introduced. In addition, an associated dataset is explained. Finally, the two proposed approaches are evaluated using a dataset.

\section{Related work}

Wang et al. [36] used AlexNet, GoogleNet, VGG-16, and ResNet=50 deep convolutional neural networks. The authors generated these models by retained ImageNet models on chest X-ray models. This work provided the classification of eight thorax diseases. The findings indicated that the ResNet-50 deep learning model outperformed all other deep learning models.

Yao et al. [38] proposed a multi-resolution based multi deep learning model. The authors used the ResNet model to reduce chest X-ray images and then they used a DensNet model to classify chest X-ray images in different resolutions. Wang and Xia [37] used classification and an attention-based deep learning model. The authors named this model the ChestNet model. The classification part of the ChestNet model contained a ResNet-152 convolutional neural network model. The output of the classification map of this model was further modelled using convolutions for more accurate classification. This work compared the proposed model with the classic ResNet model on a ChestX-ray14 dataset. The performance evaluation of the Chestnet model showed that Chestnet outperformed the ResNet model.

Gundel et al. [5] used a DensNet-121 convolutional neural network for the classification of 12 chest X-ray images. The authors created this network by adapting the ImageNet pretrained DensNet-121 model to chest images. The proposed method provided higher overall accuracy than the AlexNet, GoogleNet, VGG-16, and ResNet=50 deep networks.

Rajpurkar et al. [15] applied a DensNet-121 convolutional neural network for the detection of pneumonia. The authors also used this model for the detection of twelve thorax diseases. The authors trained the DensNet-121 model using a Chest X-ray14 dataset [36]. 
Li et al. [14] utilized an attention-based ResNet convolutional neural network. Their approach applied a U-Net deep learning segmentation model and then local regions of the X-ray images were classified using the ResNet model. Kermany [12] et al. proposed transfer learning based convolutional neural networks for Pneumonia detection. Varshni [35] et al. combined a DenseNet-169 model and support vector machines (SVM). Chest X-ray appearances are represented and related feature vectors are extracted using DenseNet-169 deep networks. The image representations are obtained by passing the architecture consisting of 169 layers. Then, the image appearances are classified using SVM for healthy and pneumonia images.

Ayan and Unver [1] utilized Xception and VGG-16 deep networks for the classification of pneumonia and healthy X-ray images. Their approach was based on retraining previously existing models for X-ray images. In other words, they used transfer learning-based models for the detection of pneumonia among pneumonia and healthy X-ray images. The VGG-16 model outperformed the Xception model for the classification task.

\section{The proposed methods}

Figures 2 and 3 present the proposed AlexNet and VGGNet16 deep learning models in conjunction with support vector machines. Classic deep learning models utilize Soft-max function for the classification of the images. In this work, we modify the deep model architectures and replace the Soft-max function with a multi-class support vector machines classifier, [2, 34]. SVM builds on margin-based loss minimization. The SVM model also employs regularization during modelling of the data samples. Therefore, the SVM model provides more accurate sample classification than the Soft-max based model. As a result, the proposed deep learning architecture employs feature extraction and then these models utilize powerful multi-class support vector machines to classify lung disease. The two proposed models allow twelve lung disease to be classified.

\subsection{Image processing}

The chest X-ray images are RGB images, and these image sizes are 256x256. The model extracts $224 \times 224 \times 3$ random image patches. Then, these image patches are used as inputs to the proposed multi-class support vector machine based AlexNet and VGGNet16 models.

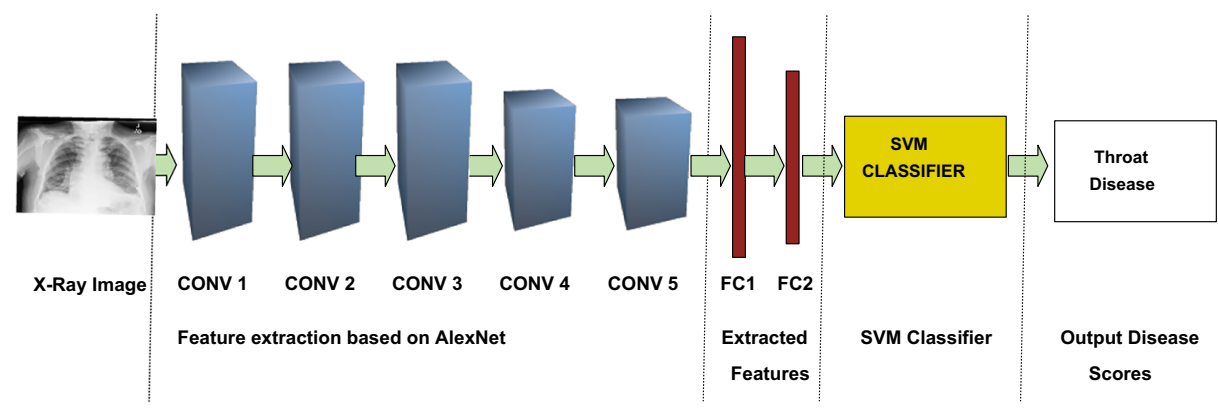

Fig. 2 The proposed SVM-AlexNet Method 


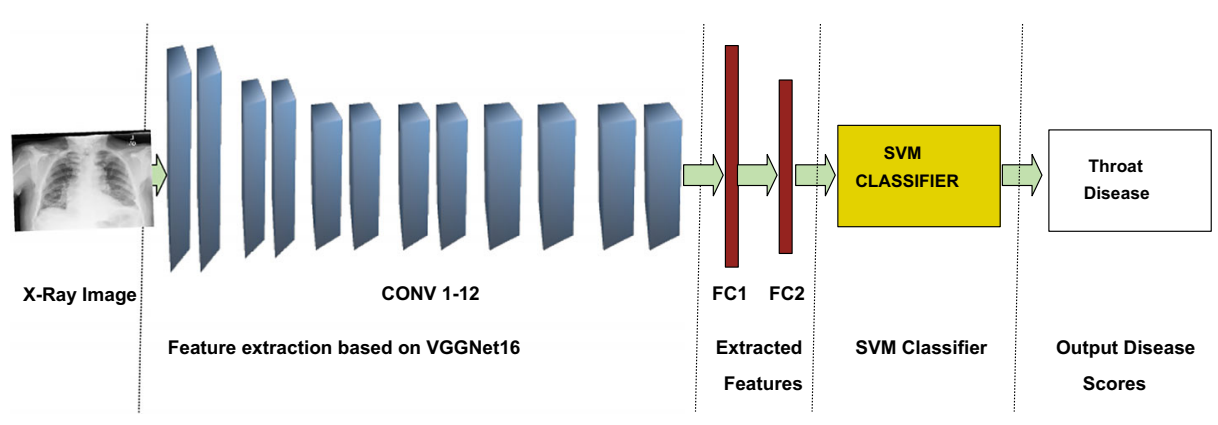

Fig. 3 The proposed SVM-VGGNet16 Method

\subsection{The proposed SVM-AlexNet method}

\subsubsection{Architecture}

Figure 2 shows the proposed SVM-AlexNet architecture. This architecture consists of five convolution layers (Conv1, Conv2, Conv3, Conv4, and Conv5), two fully connected layers (FC1 and FC2) and a multi-class support vector machine model (SVM). The proposed method utilizes five convolutional layers to extract features of chest X-ray images. Then, these features are classified into twelve throat diseases using a multi-class SVM.

\subsubsection{Modelling}

We use a set of chest X-ray images as input to the AlexNet network. Input images are convolved with 96 filters with a size of 11x11 in the first convolution layer. Subsequently, the output of the first convolutional layer is used as the input for the second convolutional layer. Then, the pooling is performed on the production of the second convolutional layer. This input is filtered with 256 kernels of size $5 \times 5$ in the second convolutional layer. This convolution process is done for the third, fourth and fifth convolutional layers. Then, fully connected layers are constructed.

\subsubsection{Multi-class support vector machines}

We used multi-class support vector machines to map feature vectors of the convolutional layers to lung disease [7, 8]. We used a one-vs-rest approach to obtain multi-class support vector machines. There are mainly one-versus-one and one-versus-rest approaches for extending SVM to multi-class. We used the one-vs-rest approach since it is simple to use and computationally less expensive.

We trained the proposed multi-class support vector machines as follows. All chest X-ray images are passed through the trained AlexNet model, and corresponding feature vectors of the chest X-ray images are obtained. These feature vectors are retrieved from the FC2 layer of the trained Alexnet model. The dimension of these feature vectors is $1 \mathrm{x} 4096$. Then, these feature vectors are fed into the multi-class support vector machines for classifying twelve throat disease. Section 3.4 describes support vector machines for binary classification. 


\subsection{The proposed SVM-VGGNet16 method}

\subsubsection{Architecture}

Figure 3 shows the proposed SVM-VGGNet16 architecture. This architecture consists of thirteen convolution layers (Conv1-Conv13), two fully connected layers (FC1-FC2) and a multi-class support vector machine model (SVM). The proposed method employs thirteen convolutional layers to model and extract features of chest X-ray images. Then, the extracted features are classified into 12 throat diseases using multi-class SVM in Section 3.2.3. The thirteen convolutional layers are responsible for the representation of chest X-ray images together with two fully connected layers (FC1 and FC2).

\subsubsection{Modelling}

First, we train the VGGNet6 network using chest X-ray images to create a model. During training, input images go through twelve convolutional layers and then three fully connected layers. Twelve convolutional layers are generated using filter sizes of $3 \times 3$. However, the number of filters is different for each of the convolution layers. The number of filters used in the first and second convolutional layer is 64. Furthermore, the third and fourth layers are obtained using 128 filters. Moreover, the fifth and size convolutional layers utilize 256. Then 512 filters are used for the last size layers.

\subsubsection{Multi-class support vector machines}

We use training images of chest X-ray images as input for the thirteen convolution layers and two fully connected layers. Then, we obtain 4096 dimensional vectors from the FC2 layer of the model. These vectors are used as inputs for the multi-class support vector machines to predict twelve lung diseases.

\subsection{Support vector machine}

The appearances of chest X-ray image deformations in Sections 3.2 and 3.3 are obtained using AlexNet and VGG-16 deep networks and then classified using support vector machines. Chest $\mathrm{X}$-ray images appearances are denoted by $\mathbf{x}_{\mathbf{1}}, \ldots \mathbf{x}_{\mathbf{n}}$ and corresponding chest $\mathrm{X}$-ray types are denoted by $\mathbf{y}_{\mathbf{1}}, \ldots \mathbf{y}_{\mathbf{n}}$. After, given a chest X-ray appearance $\mathbf{x}$, the corresponding chest X-ray image's name is predicted using support vector machines, [2, 34],

$$
f(x)=\mathbf{w} \cdot \mathbf{x}+b
$$

where $\mathrm{f}(\mathrm{x})$ denotes the estimated chest X-ray image. Weights and bias are also denoted by $w, b$, respectively. The weight and bias values are estimated by optimizing the following optimization equation.

$$
\begin{gathered}
\min _{w, b, \xi} \quad \frac{1}{2} w w^{T}+C \Sigma_{i=1}^{n} \xi_{i} \\
\text { subject to }\left\{\begin{array}{l}
y_{i}\left(w^{T} \phi\left(x_{i}\right)+b\right) \geq 1-\xi_{i} \\
\xi_{i} \geq 0, i=1, \ldots ., n
\end{array}\right.
\end{gathered}
$$

$\phi\left(x_{i}\right)$ transfers data points to other dimensions. Slack variables are denoted by $\xi_{i}$, and these variables guide observations towards the margin. $\mathrm{C}$ defines regularization. 


\section{The assumed dataset and performance metrics}

ChestX-ray14 dataset, [36], is one of the largest datasets related to chest X-ray images. This database contains fourteen thorax disease. Table 1 shows fourteen thorax disease and the corresponding number of images for each of the diseases. The proposed SVM based AlexNet and VGG-16 deep models are evaluated for fourteen thorax diseases using this database.

We use accuracy, sensitivity, and specificity as performance evaluation metrics. These metrics can be described as follows.

$$
\begin{gathered}
\text { Accuracy }=\frac{\mathrm{TP}+\mathrm{TN}}{\mathrm{TP}+\mathrm{TN}+\mathrm{FP}+\mathrm{FN}} \\
\text { Sensitivity }=\frac{\mathrm{TP}}{\mathrm{TP}+\mathrm{FN}} \\
\text { Specificity }=\frac{\mathrm{TN}}{\mathrm{TN}+\mathrm{FP}}
\end{gathered}
$$

We denote true positive, positive, true negative, false positive, and false negative as TP, TN, $\mathrm{FP}$, and FN, respectively.

\section{Performance evaluation}

We report the performance results of the proposed methods for twelve chest diseases in Table 3. Table 3 shows the area under the curve values (AUC).

We trained proposed methods using $70 \%$ of the ChestX-ray dataset, and we evaluated the network performances using $40 \%$ of the chest X-ray dataset.

\subsection{Evaluation of The proposed models}

Table 2 shows the accuracy, sensitivity and specificity values of proposed methods and classic deep learning methods. The proposed AlexNet+SVM and VGG16+SVM methods provide higher accuracy values that classic AlexNet and VGG16+SVM methods.

Figure 4 (a),(b), (c), and (d) also show confusion matrices of the AlexNet, VGG-16, and SVM based AlexNet and VGG-16 models. These matrices show both the number of true positive disease detections and accuracy values of the twelve chest disease. The overall accuracy values of the AlexNet, VGG-16, AlexNet+SVM, and VGG-16+SVM models are

Table 1 Thorax diseases and number of images

\begin{tabular}{ll}
\hline Thorax disease & No. of Images \\
\hline Atelectasis & 11167 \\
Cardiomegaly & 12071 \\
Effusion & 7646 \\
Infiltration & 16316 \\
Mass & 10042 \\
Nodule & 6480 \\
Pneumonia & 9836 \\
Pneumothorax & 4693 \\
Consolidation & 4544 \\
Edema & 8875 \\
Emphysema & 10070 \\
Fibrosis & 10380 \\
\hline
\end{tabular}


Table 2 The accuracy, sensitivity and specificity values of our proposed methods

AlexNet+SVM AC value (0.96) is bold in Table 2

VGG16+SVM AC value (0.98) is bold Table 2

\begin{tabular}{llll}
\hline Proposed Method & AC & SE & SP \\
\hline AlexNet & 0.94 & 0.99 & 0.94 \\
VGG16 & 0.95 & 0.96 & 0.95 \\
AlexNet+SVM & $\mathbf{0 . 9 6}$ & 0.96 & 0.96 \\
VGG16+SVM & $\mathbf{0 . 9 8}$ & 0.99 & 0.98 \\
\hline
\end{tabular}

$94.1 \%, 95.3 \%, 96.3 \%$, and 98.1\%. The proposed AlexNet+SVM and VGG-16+SVM models provide higher accuracy than classic deep learning models. Moreover, the AlexNet-based SVM provides an average AUC value of $98 \%$ and the VGGNet-based SVM provides an average AUC value of $97 \%$ for twelve chest X-ray diseases.

\subsection{Comparison of the proposed method and other methods}

Table 3 shows the performances for AlexNet, AlexNet+SVM, VGG-16, and VGG-16+SVM models. The AUC values of the models are similar.

AlexNet, GoogleNet, VGG-16, ResNet-50, DensNet, and attention-based deep learningbased methods were utilised for the classification of X-ray images. Previous performance results have shown that the ResNet-50 deep network provides higher accuracy than AlexNet, GoogleNet, and VGG-16 [36]. More recent studies show that DensNet architecture [15] further improves the performance of the chest X-ray image classification. The proposed support vector machine-based deep network has shown that combining AlexNet and VGG-16 networks with support vector machines improve the classic AlexNet and VGG-16 networks. The performance results indicate that classifying chest X-ray images using SVM instead of the Softmax classifier results in a more powerful learning algorithm.

\section{Discussion}

\subsection{Accuracy}

The proposed methods outperform other techniques for the classification of the twelve throat disease. Previous works have utilized deep learning methods with the Softmax function for classification. In contrast, the proposed approaches employ deep learning models in conjunction with support vector machines (SVMs) for classification. Softmax is based on cross-entropy minimization while the data samples are classified. On the other hand, an SVM builds on margin-based loss minimization. The SVM model also employs regularization during modelling of the data samples. Therefore, the proposed SVM provides a more accurate sample classification than the use of only cross-entropy loss minimization. Thus, the proposed approaches outperform current works for the detection of twelve throat diseases.

\subsection{Computational complexities}

Rajpurkar et al. [15] employed DensNet-121 and Wang et al., [37] use ResNet-152 deep networks. These networks convolve images to create 121 and 151 convolutional layers, respectively. In contrast, the proposed AlexNet and VGGNet16 convolve images to create 
a

\begin{tabular}{|c|c|c|c|c|c|c|c|c|c|c|c|c|c|}
\hline Mass & $\begin{array}{l}481 \\
8.0 \%\end{array}$ & $\begin{array}{c}0 \\
0.0 \%\end{array}$ & $\begin{array}{c}0 \\
0.0 \%\end{array}$ & $\begin{array}{l}25 \\
0.4 \%\end{array}$ & $\begin{array}{c}0 \\
0.0 \%\end{array}$ & $\begin{array}{c}0 \\
0.0 \%\end{array}$ & $\begin{array}{c}0 \\
0.0 \%\end{array}$ & $\begin{array}{c}0 \\
0.0 \%\end{array}$ & $\begin{array}{c}0 \\
0.0 \%\end{array}$ & $\begin{array}{l}7 \\
0.1 \%\end{array}$ & $\begin{array}{c}0 \\
0.0 \%\end{array}$ & $\begin{array}{c}0 \\
0.0 \%\end{array}$ & $\begin{array}{c}93.8 \% \\
6.2 \%\end{array}$ \\
\hline Pneumonia & $\begin{array}{c}0 \\
0.0 \%\end{array}$ & $\begin{array}{c}28 \\
0.5 \%\end{array}$ & $\begin{array}{c}0 \\
0.0 \%\end{array}$ & $\begin{array}{l}475 \\
7.9 \%\end{array}$ & $\begin{array}{c}0 \\
0.0 \%\end{array}$ & $\begin{array}{c}7 \\
0.1 \%\end{array}$ & $\begin{array}{c}0 \\
0.0 \%\end{array}$ & $\begin{array}{c}0 \\
0.0 \%\end{array}$ & $\begin{array}{c}0 \\
0.0 \%\end{array}$ & $\begin{array}{c}0 \\
0.0 \%\end{array}$ & $\begin{array}{c}0 \\
0.0 \%\end{array}$ & $\begin{array}{c}0 \\
0.0 \%\end{array}$ & $\begin{array}{c}93.1 \% \\
6.9 \%\end{array}$ \\
\hline Cardiomegaly & $\begin{array}{c}0 \\
0.0 \%\end{array}$ & $\begin{array}{c}0 \\
0.0 \%\end{array}$ & $\begin{array}{r}114 \\
1.9 \%\end{array}$ & $\begin{array}{c}0 \\
0.0 \%\end{array}$ & $\begin{array}{l}489 \\
8.2 \%\end{array}$ & $\begin{array}{c}0 \\
0.0 \%\end{array}$ & $\begin{array}{c}0 \\
0.0 \%\end{array}$ & $\begin{array}{c}0 \\
0.0 \%\end{array}$ & $\begin{array}{c}0 \\
0.0 \%\end{array}$ & $\begin{array}{c}0 \\
0.0 \%\end{array}$ & $\begin{array}{c}0 \\
0.0 \%\end{array}$ & $\begin{array}{c}22 \\
0.4 \%\end{array}$ & $\begin{array}{l}78.2 \% \\
21.8 \%\end{array}$ \\
\hline Nodule & $\begin{array}{c}0 \\
0.0 \%\end{array}$ & $\begin{array}{c}0 \\
0.0 \%\end{array}$ & $\begin{array}{c}0 \\
0.0 \%\end{array}$ & $\begin{array}{c}0 \\
0.0 \%\end{array}$ & $\begin{array}{c}0 \\
0.0 \%\end{array}$ & $\begin{array}{c}0 \\
0.0 \%\end{array}$ & $\begin{array}{l}491 \\
8.2 \%\end{array}$ & $\begin{array}{c}0 \\
0.0 \%\end{array}$ & $\begin{array}{c}13 \\
0.2 \%\end{array}$ & $\begin{array}{c}0 \\
0.0 \%\end{array}$ & $\begin{array}{c}0 \\
0.0 \%\end{array}$ & $\begin{array}{c}0 \\
0.0 \%\end{array}$ & $\begin{array}{c}97.4 \% \\
2.6 \%\end{array}$ \\
\hline Pneumothorax & $\begin{array}{c}0 \\
0.0 \%\end{array}$ & $\begin{array}{c}0 \\
0.0 \%\end{array}$ & $\begin{array}{c}0 \\
0.0 \%\end{array}$ & $\begin{array}{c}0 \\
0.0 \%\end{array}$ & $\begin{array}{c}0 \\
0.0 \%\end{array}$ & $\begin{array}{c}0 \\
0.0 \%\end{array}$ & $\begin{array}{c}0 \\
0.0 \%\end{array}$ & $\begin{array}{l}483 \\
8.1 \%\end{array}$ & $\begin{array}{c}0 \\
0.0 \%\end{array}$ & $\begin{array}{c}0 \\
0.0 \%\end{array}$ & $\begin{array}{c}0 \\
0.0 \%\end{array}$ & $\begin{array}{c}0 \\
0.0 \%\end{array}$ & $\begin{array}{l}100 \% \\
0.0 \%\end{array}$ \\
\hline Consolidation & $\begin{array}{c}0 \\
0.0 \%\end{array}$ & $\begin{array}{c}0 \\
0.0 \%\end{array}$ & $\begin{array}{c}0 \\
0.0 \%\end{array}$ & $\begin{array}{c}0 \\
0.0 \%\end{array}$ & $\begin{array}{c}0 \\
0.0 \%\end{array}$ & $\begin{array}{c}0 \\
0.0 \%\end{array}$ & $\begin{array}{c}0 \\
0.0 \%\end{array}$ & $\begin{array}{c}0 \\
0.0 \%\end{array}$ & $\begin{array}{l}487 \\
8.1 \%\end{array}$ & $\begin{array}{c}0 \\
0.0 \%\end{array}$ & $\begin{array}{c}0 \\
0.0 \%\end{array}$ & $\begin{array}{c}0 \\
0.0 \%\end{array}$ & $\begin{array}{l}100 \% \\
0.0 \%\end{array}$ \\
\hline Fibrosis & $\begin{array}{c}0 \\
0.0 \%\end{array}$ & $\begin{array}{c}0 \\
0.0 \%\end{array}$ & $\begin{array}{c}0 \\
0.0 \%\end{array}$ & $\begin{array}{c}0 \\
0.0 \%\end{array}$ & $\begin{array}{c}0 \\
0.0 \%\end{array}$ & $\begin{array}{c}0 \\
0.0 \%\end{array}$ & $\begin{array}{c}0 \\
0.0 \%\end{array}$ & $\begin{array}{c}0 \\
0.0 \%\end{array}$ & $\begin{array}{c}0 \\
0.0 \%\end{array}$ & $\begin{array}{c}0 \\
0.0 \%\end{array}$ & $\begin{array}{c}0 \\
0.0 \%\end{array}$ & $\begin{array}{l}468 \\
7.8 \%\end{array}$ & $\begin{array}{l}100 \% \\
0.0 \%\end{array}$ \\
\hline & $\begin{array}{l}96.2 \% \\
3.8 \%\end{array}$ & $\begin{array}{l}94.4 \% \\
5.6 \%\end{array}$ & $\begin{array}{l}63.8 \% \\
36.2 \%\end{array}$ & $\begin{array}{l}95.0 \% \\
5.0 \%\end{array}$ & $\begin{array}{l}97.8 \% \\
2.2 \%\end{array}$ & $\begin{array}{l}98.6 \% \\
140 \%\end{array}$ & $\begin{array}{l}98.2 \% \\
1.8 \%\end{array}$ & $\begin{array}{l}96.6 \% \\
3.4 \%\end{array}$ & $\begin{array}{l}97.4 \% \\
2.6 \%\end{array}$ & $\begin{array}{l}98.6 \% \\
1.4 \%\end{array}$ & $\begin{array}{c}98.8 \\
1.2 \%\end{array}$ & $\begin{array}{l}93.6 \% \\
6.4 \%\end{array}$ & $\begin{array}{c}94.1 \% \\
5.9 \%\end{array}$ \\
\hline
\end{tabular}

b

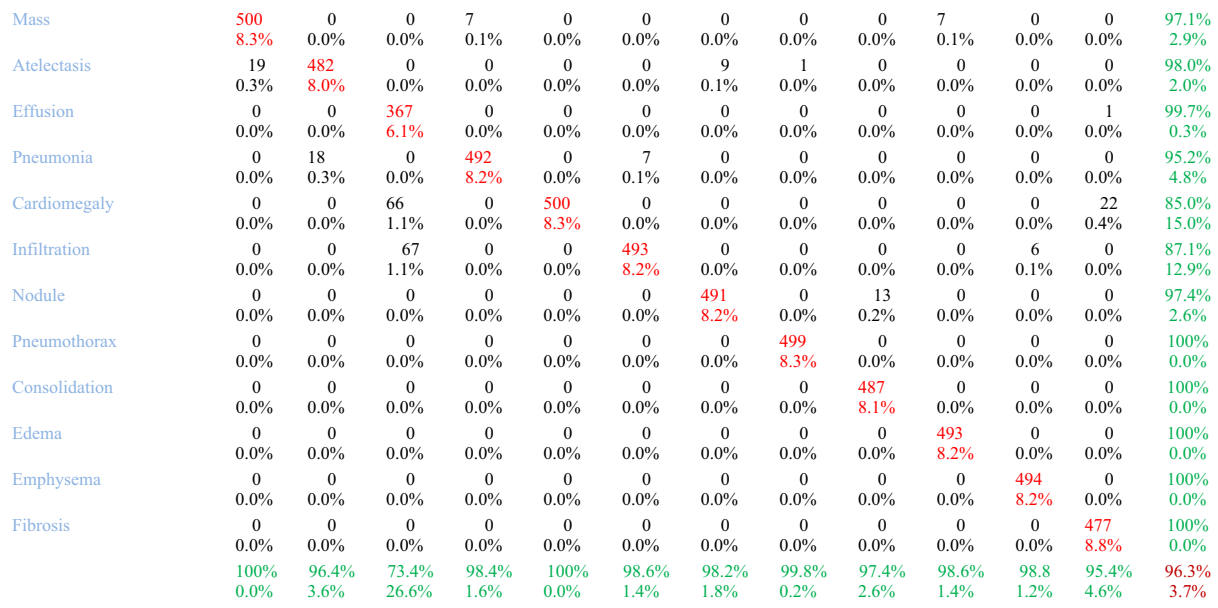

Fig. 4 Chest X-ray image in ChestX-ray8 dataset 
c

\begin{tabular}{|c|c|c|c|c|c|c|c|c|c|c|c|c|c|}
\hline Mass & $\begin{array}{l}481 \\
8.0 \%\end{array}$ & $\begin{array}{c}0 \\
0.0 \%\end{array}$ & $\begin{array}{c}0 \\
0.0 \%\end{array}$ & $\begin{array}{l}11 \\
0.2 \%\end{array}$ & $\begin{array}{c}0 \\
0.0 \%\end{array}$ & $\begin{array}{c}0 \\
0.0 \%\end{array}$ & $\begin{array}{c}0 \\
0.0 \%\end{array}$ & $\begin{array}{c}0 \\
0.0 \%\end{array}$ & $\begin{array}{c}0 \\
0.0 \%\end{array}$ & $\begin{array}{l}7 \\
0.1 \%\end{array}$ & $\begin{array}{c}0 \\
0.0 \%\end{array}$ & $\begin{array}{c}0 \\
0.0 \%\end{array}$ & $\begin{array}{c}96.4 \% \\
3.2 \%\end{array}$ \\
\hline Atelectasis & $\begin{array}{r}19 \\
0.3 \%\end{array}$ & $\begin{array}{l}472 \\
7.9 \%\end{array}$ & $\begin{array}{c}0 \\
0.0 \%\end{array}$ & $\begin{array}{c}0 \\
0.0 \%\end{array}$ & $\begin{array}{c}0 \\
0.0 \%\end{array}$ & $\begin{array}{c}0 \\
0.0 \%\end{array}$ & $\begin{array}{c}9 \\
0.1 \%\end{array}$ & $\begin{array}{c}17 \\
0.3 \%\end{array}$ & $\begin{array}{c}0 \\
0.0 \%\end{array}$ & $\begin{array}{c}0 \\
0.0 \%\end{array}$ & $\begin{array}{c}0 \\
0.0 \%\end{array}$ & $\begin{array}{c}0 \\
0.0 \%\end{array}$ & $\begin{array}{c}91.3 \% \\
8.7 \%\end{array}$ \\
\hline Effusion & $\begin{array}{c}0 \\
0.0 \%\end{array}$ & $\begin{array}{c}0 \\
0.0 \%\end{array}$ & $\begin{array}{l}367 \\
6.1 \%\end{array}$ & $\begin{array}{c}0 \\
0.0 \%\end{array}$ & $\begin{array}{c}0 \\
0.0 \%\end{array}$ & $\begin{array}{c}0 \\
0.0 \%\end{array}$ & $\begin{array}{c}0 \\
0.0 \%\end{array}$ & $\begin{array}{c}0 \\
0.0 \%\end{array}$ & $\begin{array}{c}0 \\
0.0 \%\end{array}$ & $\begin{array}{c}0 \\
0.0 \%\end{array}$ & $\begin{array}{c}0 \\
0.0 \%\end{array}$ & $\begin{array}{c}1 \\
0.0 \%\end{array}$ & $\begin{array}{c}99.7 \% \\
0.3 \%\end{array}$ \\
\hline Pneumonia & $\begin{array}{c}0 \\
0.0 \%\end{array}$ & $\begin{array}{r}28 \\
0.5 \%\end{array}$ & $\begin{array}{c}0 \\
0.0 \%\end{array}$ & $\begin{array}{l}489 \\
8.2 \%\end{array}$ & $\begin{array}{c}0 \\
0.0 \%\end{array}$ & $\begin{array}{c}7 \\
0.1 \%\end{array}$ & $\begin{array}{c}0 \\
0.0 \%\end{array}$ & $\begin{array}{c}0 \\
0.0 \%\end{array}$ & $\begin{array}{c}0 \\
0.0 \%\end{array}$ & $\begin{array}{c}0 \\
0.0 \%\end{array}$ & $\begin{array}{c}0 \\
0.0 \%\end{array}$ & $\begin{array}{c}0 \\
0.0 \%\end{array}$ & $\begin{array}{c}93.3 \% \\
6.7 \%\end{array}$ \\
\hline Cardiomegaly & $\begin{array}{c}0 \\
0.0 \%\end{array}$ & $\begin{array}{c}0 \\
0.0 \%\end{array}$ & $\begin{array}{c}66 \\
1.1 \%\end{array}$ & $\begin{array}{c}0 \\
0.0 \%\end{array}$ & $\begin{array}{l}489 \\
8.2 \%\end{array}$ & $\begin{array}{c}0 \\
0.0 \%\end{array}$ & $\begin{array}{c}0 \\
0.0 \%\end{array}$ & $\begin{array}{c}0 \\
0.0 \%\end{array}$ & $\begin{array}{c}0 \\
0.0 \%\end{array}$ & $\begin{array}{c}0 \\
0.0 \%\end{array}$ & $\begin{array}{c}0 \\
0.0 \%\end{array}$ & $\begin{array}{c}22 \\
0.4 \%\end{array}$ & $\begin{array}{l}84.7 \% \\
15.3 \%\end{array}$ \\
\hline Infiltration & $\begin{array}{c}0 \\
0.0 \%\end{array}$ & $\begin{array}{c}0 \\
0.0 \%\end{array}$ & $\begin{array}{r}67 \\
1.1 \%\end{array}$ & $\begin{array}{c}0 \\
0.0 \%\end{array}$ & $\begin{array}{c}11 \\
0.2 \%\end{array}$ & $\begin{array}{l}493 \\
8.2 \%\end{array}$ & $\begin{array}{c}0 \\
0.0 \%\end{array}$ & $\begin{array}{c}0 \\
0.0 \%\end{array}$ & $\begin{array}{c}0 \\
0.0 \%\end{array}$ & $\begin{array}{c}0 \\
0.0 \%\end{array}$ & $\begin{array}{c}6 \\
0.1 \%\end{array}$ & $\begin{array}{c}0 \\
0.0 \%\end{array}$ & $\begin{array}{l}85.4 \% \\
14.6 \%\end{array}$ \\
\hline Nodule & $\begin{array}{c}0 \\
0.0 \%\end{array}$ & $\begin{array}{c}0 \\
0.0 \%\end{array}$ & $\begin{array}{c}0 \\
0.0 \%\end{array}$ & $\begin{array}{c}0 \\
0.0 \%\end{array}$ & $\begin{array}{c}0 \\
0.0 \%\end{array}$ & $\begin{array}{c}0 \\
0.0 \%\end{array}$ & $\begin{array}{l}491 \\
8.2 \%\end{array}$ & $\begin{array}{c}0 \\
0.0 \%\end{array}$ & $\begin{array}{c}13 \\
0.2 \%\end{array}$ & $\begin{array}{c}0 \\
0.0 \%\end{array}$ & $\begin{array}{c}0 \\
0.0 \%\end{array}$ & $\begin{array}{c}0 \\
0.0 \%\end{array}$ & $\begin{array}{c}97.4 \% \\
2.6 \%\end{array}$ \\
\hline Pneumothorax & $\begin{array}{c}0 \\
0.0 \%\end{array}$ & $\begin{array}{c}0 \\
0.0 \%\end{array}$ & $\begin{array}{c}0 \\
0.0 \%\end{array}$ & $\begin{array}{c}0 \\
0.0 \%\end{array}$ & $\begin{array}{c}0 \\
0.0 \%\end{array}$ & $\begin{array}{c}0 \\
0.0 \%\end{array}$ & $\begin{array}{c}0 \\
0.0 \%\end{array}$ & $\begin{array}{l}483 \\
8.1 \%\end{array}$ & $\begin{array}{c}0 \\
0.0 \%\end{array}$ & $\begin{array}{c}0 \\
0.0 \%\end{array}$ & $\begin{array}{c}0 \\
0.0 \%\end{array}$ & $\begin{array}{c}0 \\
0.0 \%\end{array}$ & $\begin{array}{l}100 \% \\
0.0 \%\end{array}$ \\
\hline Consolidation & $\begin{array}{c}0 \\
0.0 \%\end{array}$ & $\begin{array}{c}0 \\
0.0 \%\end{array}$ & $\begin{array}{c}0 \\
0.0 \%\end{array}$ & $\begin{array}{c}0 \\
0.0 \%\end{array}$ & $\begin{array}{c}0 \\
0.0 \%\end{array}$ & $\begin{array}{c}0 \\
0.0 \%\end{array}$ & $\begin{array}{c}0 \\
0.0 \%\end{array}$ & $\begin{array}{c}0 \\
0.0 \%\end{array}$ & $\begin{array}{l}487 \\
8.1 \%\end{array}$ & $\begin{array}{c}0 \\
0.0 \%\end{array}$ & $\begin{array}{c}0 \\
0.0 \%\end{array}$ & $\begin{array}{c}0 \\
0.0 \%\end{array}$ & $\begin{array}{l}100 \% \\
0.0 \%\end{array}$ \\
\hline Edema & $\begin{array}{c}0 \\
0.0 \%\end{array}$ & $\begin{array}{c}0 \\
0.0 \%\end{array}$ & $\begin{array}{c}0 \\
0.0 \%\end{array}$ & $\begin{array}{c}0 \\
0.0 \%\end{array}$ & $\begin{array}{c}0 \\
0.0 \%\end{array}$ & $\begin{array}{c}0 \\
0.0 \%\end{array}$ & $\begin{array}{c}0 \\
0.0 \%\end{array}$ & $\begin{array}{c}0 \\
0.0 \%\end{array}$ & $\begin{array}{c}0 \\
0.0 \%\end{array}$ & $\begin{array}{l}493 \\
8.2 \%\end{array}$ & $\begin{array}{c}0 \\
0.0 \%\end{array}$ & $\begin{array}{c}0 \\
0.0 \%\end{array}$ & $\begin{array}{l}100 \% \\
0.0 \%\end{array}$ \\
\hline Emphysema & $\begin{array}{c}0 \\
0.0 \%\end{array}$ & $\begin{array}{c}0 \\
0.0 \%\end{array}$ & $\begin{array}{c}0 \\
0.0 \%\end{array}$ & $\begin{array}{c}0 \\
0.0 \%\end{array}$ & $\begin{array}{c}0 \\
0.0 \%\end{array}$ & $\begin{array}{c}0 \\
0.0 \%\end{array}$ & $\begin{array}{c}0 \\
0.0 \%\end{array}$ & $\begin{array}{c}0 \\
0.0 \%\end{array}$ & $\begin{array}{c}0 \\
0.0 \%\end{array}$ & $\begin{array}{c}0 \\
0.0 \%\end{array}$ & $\begin{array}{l}494 \\
8.2 \%\end{array}$ & $\begin{array}{c}0 \\
0.0 \%\end{array}$ & $\begin{array}{l}100 \% \\
0.0 \%\end{array}$ \\
\hline Fibrosis & $\begin{array}{c}0 \\
0.0 \% \\
96.2 \% \\
3.8 \%\end{array}$ & \begin{tabular}{c}
\multicolumn{1}{c}{0} \\
$0.0 \%$ \\
$94.4 \%$ \\
$5.6 \%$
\end{tabular} & $\begin{array}{c}0 \\
0.0 \% \\
73.4 \% \\
26.6 \%\end{array}$ & $\begin{array}{c}0 \\
0.0 \% \\
97.8 \% \\
2.2 \%\end{array}$ & $\begin{array}{c}0 \\
0.0 \% \\
97.8 \% \\
2.2 \%\end{array}$ & $\begin{array}{c}0 \\
0.0 \% \\
98.6 \% \\
140 \%\end{array}$ & \begin{tabular}{c}
\multicolumn{1}{c}{0} \\
$0.0 \%$ \\
$98.2 \%$ \\
$1.8 \%$
\end{tabular} & $\begin{array}{c}c \\
0.0 \% \\
96.6 \% \\
3.4 \%\end{array}$ & \begin{tabular}{c}
\multicolumn{1}{c}{0} \\
$0.0 \%$ \\
$97.4 \%$ \\
$2.6 \%$
\end{tabular} & $\begin{array}{c}0 \\
0.0 \% \\
98.6 \% \\
1.4 \%\end{array}$ & $\begin{array}{c}0 \\
0.0 \% \\
98.8 \\
1.2 \%\end{array}$ & $\begin{array}{l}477 \\
8.0 \% \\
95.4 \% \\
4.6 \%\end{array}$ & $\begin{array}{c}100 \% \\
0.0 \% \\
95.3 \% \\
4.7 \%\end{array}$ \\
\hline
\end{tabular}

d

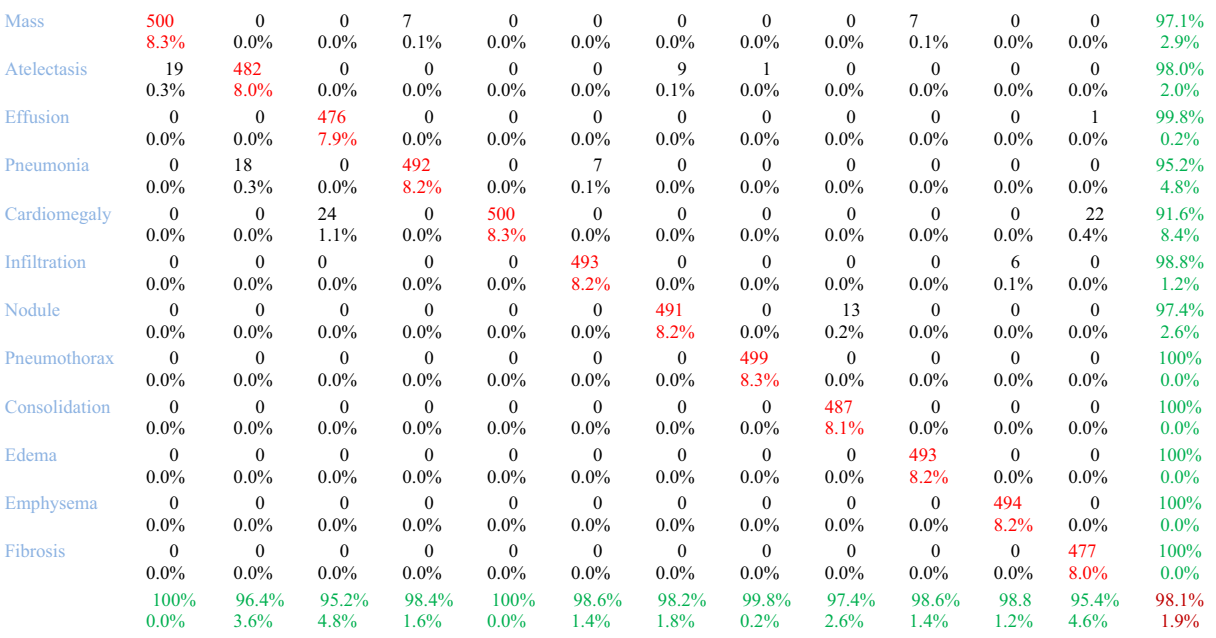

Fig. 4 (continued)

8 and 16 layers. Since the proposed methods include fewer layers than the DensNet-121 and ResNet-152 models, the models are more computationally efficient. Furthermore, novel methods employ SVM models; therefore, the models are more accurate than the DenseNet121 and ResNet-152 modes.

\subsection{AlexNet computational complexity}

Table 4 provides the number of convolution operations in each of the five convolutional layers of the AlexNet network. We also provide the total number of convolution operation for five convolutional layers. 


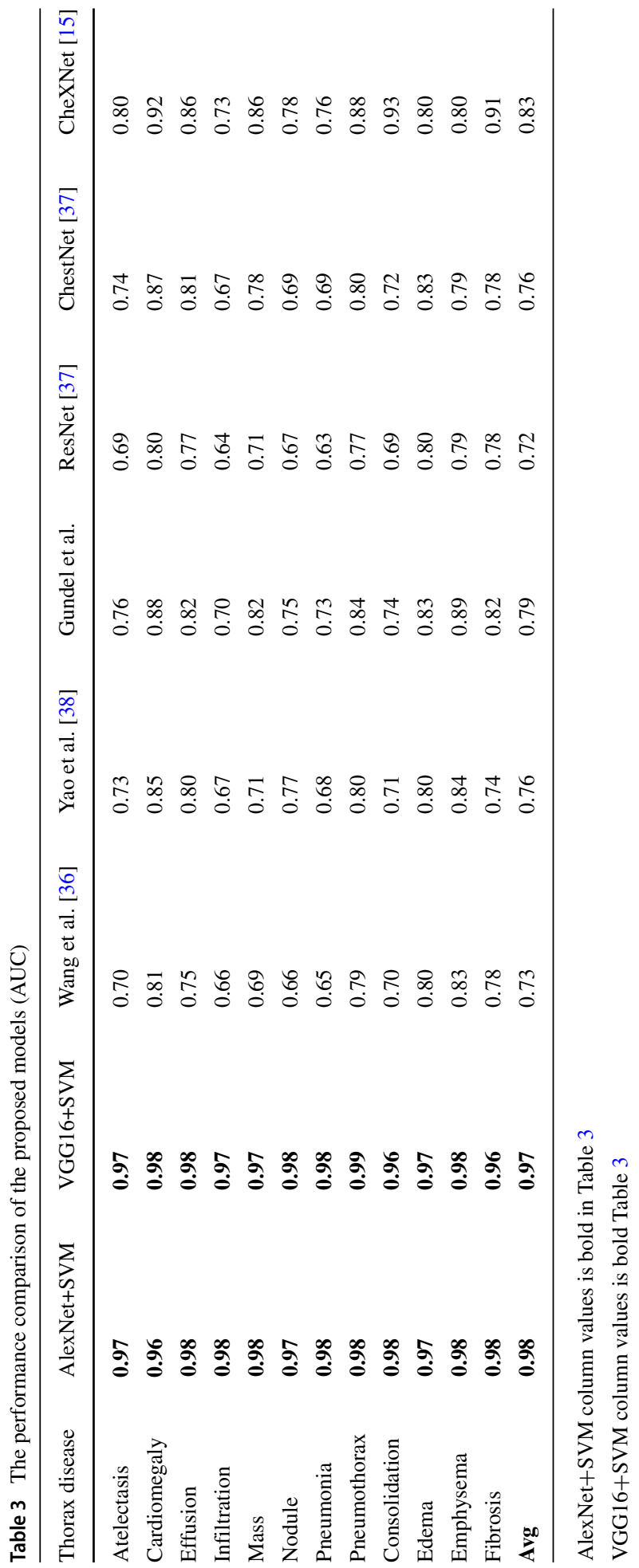


Table 4 AlexNet computational complexity

\begin{tabular}{llllll}
\hline Conv. layer & Number of filters & Filter size & Stride & Padding & Number of convolutions \\
\hline 1.Layer & 64 & $11 \times 11$ & $4 \times 4$ & $2 \times 2$ & 3472 \\
2.Layer & 192 & $5 \times 5$ & $1 \times 1$ & $2 \times 2$ & 42816 \\
3.Layer & 384 & $3 \times 3$ & $1 \times 1$ & $1 \times 1$ & 85632 \\
4.Layer & 256 & $3 \times 3$ & $1 \times 1$ & $1 \times 1$ & 57088 \\
5.Layer & 256 & $3 \times 3$ & $1 \times 1$ & $1 \times 1$ & 57088 \\
Total & - & - & - & - & 246096 \\
\hline
\end{tabular}

\subsection{VGG16 computational complexity}

Table 5 provides the number of convolution operations of thirteen convolutional layers of the VGG16 network. We also provide the total number of convolution operation for five convolutional layers.

\section{Advantages and disadvantages}

One of the advantages of the proposed methods is that a support vector machine (SVM) can easily be integrated into a deep learning architecture. The trained SVM model retrieves feature vectors from the last fully connected deep learning model, and it provides class scores. Another advantage is that using SVM instead of the Softmax classifier allows better classification accuracy for disease detection. However, the proposed methods also have certain disadvantages. Deep learning models, in conjunction with SVM models, require two training tasks. First, deep learning models are trained, and then the models are created.

Table 5 VGG16 computational complexity

\begin{tabular}{llllll}
\hline Conv. Layer & Number of filters & Filter size & Stride & Padding & Number of convolutions \\
\hline 1.Layer & 64 & $3 \times 3$ & $1 \times 1$ & $1 \times 1$ & 14272 \\
2.Layer & 64 & $3 \times 3$ & $1 \times 1$ & $1 \times 1$ & 14272 \\
3.Layer & 128 & $3 \times 3$ & $1 \times 1$ & $1 \times 1$ & 28544 \\
4.Layer & 128 & $3 \times 3$ & $1 \times 1$ & $1 \times 1$ & 28544 \\
5.Layer & 256 & $3 \times 3$ & $1 \times 1$ & $1 \times 1$ & 57088 \\
6.Layer & 256 & $3 \times 3$ & $1 \times 1$ & $1 \times 1$ & 57088 \\
7.Layer & 256 & $3 \times 3$ & $1 \times 1$ & $1 \times 1$ & 57088 \\
8.Layer & 512 & $3 \times 3$ & $1 \times 1$ & $1 \times 1$ & 114176 \\
9.Layer & 512 & $3 \times 3$ & $1 \times 1$ & $1 \times 1$ & 114176 \\
10.Layer & 512 & $3 \times 3$ & $1 \times 1$ & $1 \times 1$ & 114176 \\
11.Layer & 512 & $3 \times 3$ & $1 \times 1$ & $1 \times 1$ & 114176 \\
12.Layer & 512 & $3 \times 3$ & $1 \times 1$ & $1 \times 1$ & 114176 \\
13.Layer & 512 & $3 \times 3$ & $1 \times 1$ & $1 \times 1$ & 114176 \\
Total & - & - & - & - & 941952 \\
\hline
\end{tabular}


The generated models are used to extract features from the images. Second, extracted features are used as inputs to the SVM model for SVM model training. However, classic deep networks are only required in the training step.

\section{Limitations}

We only use one dataset to compare the proposed methods with other methods. Using one dataset might not show whether the models are generalizable or not. Models can be tested on more than one dataset to evaluate them in terms of generalizability. Another limitation is that running models and SVM models might be problematic for mobile phones or web servers. The reason is that the available hardware memory capacity of these devices is limited.

\section{Conclusion}

This study presents two new support vector machines based on AlexNet and VGG-16 deep learning models for the classification of chest X-ray images. First, AlexNet architecture is proposed to model skin image appearances, and then support vector machines are used to classify these appearances. This method is utilized to support vector machines instead of the Softmax method for more robust and accurate chest X-ray image classification. Then, we developed another support vector machine-based VGG-16 deep learning method. Similarly, this method convolves images for extracted features and classifies skin lesions using support vector machines. The proposed methodologies replace the Softmax layer with SVM. Since support vector machines are more powerful classifiers than Softmax, the proposed methods provide higher accuracy compared to classic methods. The performance results show that the proposed models outperform the typical deep learning AlexNet and VGG-16 networks.

Funding The authors did not receive any funding for this work.

\section{Declarations}

Conflict of Interests The author of this paper declares that there is no conflict of interest regarding the publication of this paper.

\section{References}

1. Ayan E, Ünver H. M. (2019) Diagnosis of pneumonia from chest x-ray images using deep learning. In: 2019 scientific meeting on electrical-electronics biomedical engineering and computer science (EBBT). pp 1-5

2. Burges CJC (1998) A tutorial on support vector machines for pattern recognition. Data Min Knowl Discov 2:121-167

3. Deng J, Dong W, Socher R, Li L-J, Li K, Fei-Fei L (2009) ImageNet: A large-scale hierarchical image database. In: Proceedings of the IEEE conference on computer vision and pattern recognition

4. Garstka J, Strzelecki M (2020) Pneumonia detection in x-ray chest images based on convolutional neural networks and data augmentation methods. In: 2020 Signal processing: algorithms, architectures, arrangements, and applications (SPA), pp 18-23

5. Guendel S, Grbic S, Georgescu B, Zhou K, Ritschl L, Meier A, Comaniciu D (2018) Learning to recognize abnormalities in chest X-rays with location-aware dense networks. arXiv:1803.04565 
6. He K, Zhang X, Ren S, Sun J (2016) Deep residual learning for image recognition. In: Proceedings of the IEEE conference on computer vision and pattern recognition, pp 770-778

7. Hsu C-W, Lin C-J (1998) Multi-class support vector machines. Technical Report

8. Hsu C-W, Lin C-J (2002) A comparison of methods for multiclass support vector machines. IEEE Trans Neural Netw 13:415-425

9. Huang G, Liu Z, Maaten L, Weinberger K (2016) Densely connected convolutional networks

10. Kaymak S, Esmaili P, Serener A (2018) Deep learning for two-step classification of malignant pigmented skin lesions. In: 14th Symposium on neural networks and applications (NEUREL). IEEE, pp 1-6

11. Kaymak S, Serener A (2018) Automated age-related macular degeneration and diabetic macular edema detection on oct images using deep learning. In: IEEE 14th international conference on intelligent computer communication and processing (ICCP). IEEE, pp 265-269

12. Kermany DS, Goldbaum M, Cai W, Valentim CC, Liang H, Baxter SL, McKeown A, Yang G, Wu X, Yan F, Dong J, Prasadha MK, Pei J, Ting MY, Zhu J, Li C, Hewett S, Dong J, Ziyar I, Shi A, Zhang R, Zheng L, Hou R, Shi W, Fu X, Duan Y, Huu VA, Wen C, Zhang ED, Zhang CL, Li O, Wang X, Singer MA, Sun X, Xu J, Tafreshi A, Lewis MA, Xia H, Zhang K (2018) Identifying medical diagnoses and treatable diseases by image-based deep learning. Cell 172:1122-1131.e9

13. Krizhevsky A, Sutskever I, Hinton GE (2017) Imagenet classification with deep convolutional neural networks. Commun ACM 60:84-90

14. Li B, Kang G, Cheng K, Zhang N (2019) Attention-guided convolutional neural network for detecting pneumonia on chest X-rays. In: 2019 41st annual international conference of the ieee engineering in medicine and biology society (EMBC), pp 4851-4854

15. Rajpurkar P, Irvin J, Zhu K, Yang B, Mehta H, Duan T, Ding D, Bagul A, Langlotz C, Shpanskaya K, Lungren MP, Ng AY (2017) Chexnet, Radiologist-level pneumonia detection on chest X-rays with deep learning. arXiv: 1711.05225

16. Serener A, Serte S (2019) Transfer learning for early and advanced glaucoma detection with convolutional neural networks. In: 2019 Medical technologies congress (TIPTEKNO), pp 1-4

17. Serener A, Serte S (2019) Dry and wet age-related macular degeneration classification using oct images and deep learning. In: Scientific meeting on electrical-electronics biomedical engineering and computer science (EBBT), pp 1-4

18. Serener A, Serte S (2019) Dry and wet age-related macular degeneration classification using oct images and deep learning. In: Scientific meeting on electrical-electronics \& biomedical engineering and computer science. IEEE, pp 1-4

19. Serener A, Serte S (2019) Transfer learning for early and advanced glaucoma detection with convolutional neural networks. In: 2019 Medical technologies congress

20. Serener A, Serte S (2020) Deep learning to distinguish covid-19 from other lung infections, pleural diseases, and lung tumors. In: Medical technologies congress (TIPTEKNO)

21. Serener A, Serte S (2020) Deep learning for mycoplasma pneumonia discrimination from pneumonias like covid-19. In: 2020 4th International symposium on multidisciplinary studies and innovative technologies (ISMSIT)

22. Serener A, Serte S (2020) Geographic variation and ethnicity in diabetic retinopathy detection via deeplearning. Turk J Electr Eng Comput Sci 28:664-678

23. Serte S, Demirel H (2019a) Gabor wavelet-based deep learning for skin lesion classification. Comput Biol Med 113:103423

24. Serte S, Demirel H (2019b) Wavelet-based deep learning for skin lesion classification. IET Image Process

25. Serte S, Demirel H (2021) Deep learning for diagnosis of covid-19 using 3d ct scans. Comput Biol Med :104306

26. Serte S, Serener A (2019) A generalized deep learning model for glaucoma detection. In: 2019 3rd International symposium on multidisciplinary studies and innovative technologies (ISMSIT), pp 1-5

27. Serte S, Serener A (2020) Early pleural effusion detection from respiratory diseases including covid-19 via deep learning. In: Medical technologies congress (TIPTEKNO)

28. Serte S, Serener A (2020) Discerning covid-19 from mycoplasma and viral pneumonia on ct images via deep learning. In: 2020 4th international symposium on multidisciplinary studies and innovative technologies (ISMSIT), pp 1-5

29. Serte S, Serener A (2021) Graph-based saliency and ensembles of convolutional neural networks for glaucoma detection. IET Image Process 15:797-804

30. Serte S, Serener A, Al-Turjman F (2020) Deep learning in medical imaging: a brief review. Trans Emerging Telecommun Technol

31. Simonyan K, Zisserman A (2014) Very deep convolutional networks for large-scale image recognition 
32. Szegedy C, Liu W, Jia Y, Sermanet P, Reed S, Anguelov D, Erhan D, Vanhoucke V, Rabinovich A (2015a) Going deeper with convolutions

33. Szegedy C, Vanhoucke V, Ioffe S, Shlens J (2015b) Rethinking the inception architecture for computer vision

34. Tang Y (2015) Deep learning using linear support vector machines

35. Varshni D, Thakral K, Agarwal L, Nijhawan R, Mittal A (2019) Pneumonia detection using cnn based feature extraction. In: 2019 IEEE international conference on electrical, computer and communication technologies (ICECCT), pp 1-7

36. Wang X, Peng Y, Lu L, Lu Z, Bagheri M, Summers RM (2017) ChestX-ray8: Hospital-scale chest Xray database and benchmarks on weakly-supervised classification and localization of common thorax diseases. In: 2017 IEEE Conference on computer vision and pattern recognition (CVPR)

37. Wang H, Xia Y (2018) Chestnet: A deep neural network for classification of thoracic diseases on chest radiography. arXiv: 1807.03058

38. Yao L, Prosky J, Poblenz E, Covington B, Lyman K (2018) Weakly supervised medical diagnosis and localization from multiple resolutions. arXiv:1803.07703

Publisher's note Springer Nature remains neutral with regard to jurisdictional claims in published maps and institutional affiliations. 